Research Paper

\title{
Photon pair sources with controlled frequency correlation
}

\author{
Keiichi EDAMATSU ${ }^{1}$, Ryosuke SHIMIZU², Wakana UENO ${ }^{3}$, Rui-Bo $\mathrm{JIN}^{4}$, \\ Fumihiro KANEDA ${ }^{5}$, Masahiro YABUNO ${ }^{6}$, Hirofumi SUZUKI ${ }^{7}$, \\ Shigehiro NAGANO ${ }^{8}$, Atsushi SYOUJI ${ }^{9}$, and Koji SUIZU ${ }^{10}$ \\ 1,3,4,5,6,7,8,9 Research Institute of Electrical Communication, Tohoku University \\ ${ }^{2}$ PRESTO, Japan Science and Technology Agency \\ ${ }^{10}$ Graduate School of Engineering, Nagoya University
}

\begin{abstract}
Development of efficient and well-controlled nonclassical photon sources is one of the keys in the quantum information and communication technology. We present our recent activities to develop advanced sources of photon pairs having controlled frequency correlation, by use of quasi-phase matching (QPM) and extended phase-matching (EPM). First, we present the generation of polarization and frequency entangled photons using QPM having two poling periods. We also demonstrate the photon pair generation with controlled frequency correlation and its application to making heralded single photons with intrinsically pure spectrotemporal modes.
\end{abstract}

\section{KEYWORDS}

quantum information, quantum optics, entanglement, quasi-phase matching, extended phase matching

\section{Introduction}

In the development of quantum information and communication technology (QICT), there are many technical issues to be solved. Development of efficient sources of entanglement is one of such essential keys toward the QICT. Photons are the most popular and powerful method to generate and distribute the entanglement [1]. Especially, spontaneous parametric down-conversion (SPDC) is frequently used to generate entangled photons in many poof-of-principle experiments. Quasi-phase matching (QPM) using periodically poled nonlinear crystals is a powerful technique to extend the applicability of nonlinear wavelength conversion including SPDC [2]. Recently, extended phase

\footnotetext{
Received November 5, 2010; Revised December 23, 2010; Accepted December $24,2010$.

1)eda@riec.tohoku.ac.jp, ${ }^{2)}$ r-simizu@pc.uec.ac.jp,

3) ueno@quantum.riec.tohoku.ac.jp, ${ }^{4)}$ jin@quantum.riec.tohoku.ac.jp,

5) kaneda@quantum.riec.tohoku.ac.jp, ${ }^{6)}$ yabuno@quantum.riec.tohoku.ac.jp.

${ }^{8)}$ nagano-shigehiro@sei.co.jp, ${ }^{9)}$ ashohji@yamanashi.ac.jp,

10) suizu@nuee.nagoya-u.ac.jp,

DOI: 10.2201/NiiPi.2011.8.2
}

matching (EPM) also attracts attention in the entangled photon generation [3]. Such new techniques are very useful not only in the entanglement generation but also in the generation of spectrally pure single photons heralded by their sister photons [4], [5]. For instance, the linear optical quantum computing [6] and the measurement-based quantum computing [7], [8] require large number of photons that are essentially indistinguishable from each other, to carry out linear quantum gate operations and to prepare large-scale cluster states. Control of frequency correlation between photons is one of the key issues to achieve efficient generation of photon pairs that satisfy the required conditions. Here we present our recent results on the generation of photon pairs having controlled frequency correlation by use of QPM and EPM in SPDC.

\section{Entangled photon generation using two-period QPM-SPDC}

SPDC is a nonlinear optical process in which a pump light is converted into two (signal and idler) lights in a 
crystal with $\chi^{(2)}$ optical nonlinearity. From a quantum optical point of view, SPDC is a process in which twin daughter photons are produced simultaneously from a parent photon. In this process, the phase matching conditions must be fulfilled:

$$
\begin{aligned}
& \omega_{p}=\omega_{s}+\omega_{i}, \\
& k_{p}=k_{s}+k_{i},
\end{aligned}
$$

where $\omega_{p, s, i}$ and $k_{p, s, i}$ are the frequencies and wave vectors of the pump (p), signal (s), and idler (i) photons, respectively. These conditions are regarded as energy and momentum conservations of the photons concerned. As a result, the signal and idler photons have natural correlations in energy and momentum. SPDC using a natural optical nonlinear crystal has limitation in its working wavelength and efficiency because the phase matching condition depends on the natural dispersion of birefringence. QPM is the technique that compensates a deviated phase matching condition with the help of periodic modulation of the nonlinear susceptibility $\chi^{(2)}$. In ferroelectric crystals such as $\mathrm{LiNbO}_{3}, \chi^{(2)}$ can be modulated by periodic domain inversion, i.e., periodic poling. Thus, the phase matching condition (2) can be controlled by the poling period $\Lambda$ as

$$
k_{p}=k_{s}+k_{i}+m \frac{2 \pi}{\Lambda},(m: \text { integer })
$$

Periodically poled $\mathrm{LiNbO}_{3}$ (PPLN) is the most major device used for QPM. In particular, QPM-SPDC in PPLN waveguides are used for the efficient generation of entangled photons in the telecom wavelength region [2], [9], aiming at applying to quantum cryptography.

Figure 1 shows the tuning curves for type-II SPDC in PPLN as a function of the poling period at $120.0^{\circ} \mathrm{C}$, calculated using the Sellmeier equations [10], [11]. The type-II SPDC emits a pair of daughter photons with orthogonal polarizations, i.e, ordinary (o) and extraordinary (e) rays. The solid and dashed curves in Fig. 1 are the tuning curves for the o-ray and e-ray, respectively. Here, we use the configuration where o-ray and e-ray have horizontal $(\mathrm{H})$ and vertical $(\mathrm{V})$ polarizations, respectively. As shown in the figure, when $\Lambda=9.25 \mu \mathrm{m}$, the e-ray at $1510 \mathrm{~nm}$ and the o-ray at $1590 \mathrm{~nm}$ are emitted. On the contrary, when $\Lambda=9.5 \mu \mathrm{m}$, the o-ray at 1510 $\mathrm{nm}$ and the e-ray at $1590 \mathrm{~nm}$ are emitted. Therefore, the superposed emission from the two poling periods can generate non-degenerate (two-color) photon pairs in the polarization-entangled state

$$
|\Psi\rangle=\frac{1}{\sqrt{2}}\left(|\mathrm{H}\rangle_{1}|\mathrm{~V}\rangle_{2}+\mathrm{e}^{\mathrm{i} \phi}|\mathrm{V}\rangle_{1}|\mathrm{H}\rangle_{2}\right)
$$

where $|\mathrm{H}\rangle_{i}$ and $|\mathrm{V}\rangle_{i}$ represent the states for horizontal and vertical polarizations of each photon, respectively.

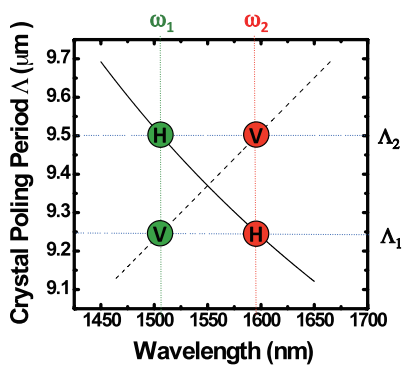

Fig. 1 Calculated tuning curve of the type-II QPM-SPDC in PPLN at $120.0^{\circ} \mathrm{C}$. The wavelength of the pump light is $\lambda p=775 \mathrm{~nm}$.

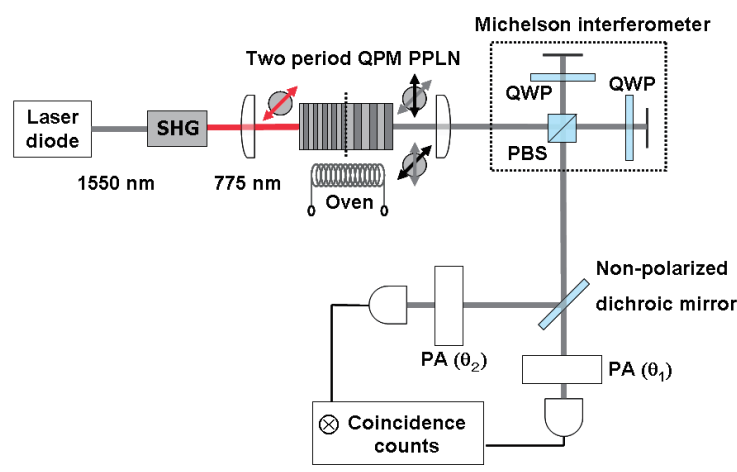

Fig. 2 Experimental setup for polarization correlation measurement of the photon pairs.

The state (4) is one of the maximally entangled states. Here, the subscripts 1 and 2 denote the photon states with wavelengths at 1510 and $1590 \mathrm{~nm}$, respectively. The phase difference $\phi$ between the two terms in (4) originates from the difference in the chromatic dispersion of the birefringent crystal for the two orthogonally polarized photons having non-degenerate frequencies. This phase difference can be controlled as described later. Since the wavelengths of photons in a pair are different from each other, one can separate the pair in a deterministic way by, for instance, using a dichroic mirror.

The experimental setup to observe the polarizationentangled photons is shown in Fig. 2. A PPLN crystal (40 $\mathrm{mm}$ long and $0.5 \mathrm{~mm}$ thick) having two poling periods was fabricated by the full-cover electrode method [12]. To produce SPDC, the crystal was pumped by the pulses at $775 \mathrm{~nm}$, which is the second harmonic of an amplified external cavity laser diode. The pulse width, repetition rate, and average power of the pump were 2.5 $\mathrm{ns}, 4 \mathrm{MHz}$, and $9 \mathrm{~mW}$, respectively. The crystal was kept at $120.0^{\circ} \mathrm{C}$ where the emission spectra generated from the two poling periods were observed to be identical having the center wavelengths at $1510 \mathrm{~nm}$ and 1590 nm. A polarization-devision Michelson interferome- 

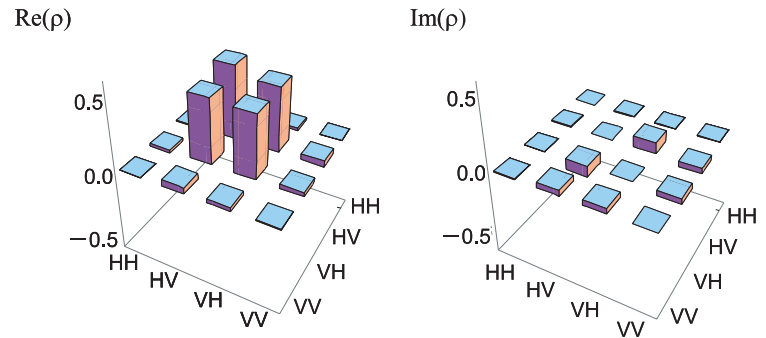

Fig. 3 Real and imaginary parts of the reconstructed density matrix $\rho$ of the two-photon polarization state.

ter compensates the group velocity difference between the photons having perpendicular polarizations. It also controls the phase difference between the two terms in (4); the phase difference $\Delta \phi$ added by the interferometer is $\Delta \phi=\left(\omega_{1}-\omega_{2}\right) \Delta L / c$, where $\Delta L$ is the path-length difference in the interferometer. Each photon split by a dichroic mirror (DM) was passed through a polarization analyzer (PA), which consists of a quarter wave plate, a half wave plate and a polarizing beam splitter. The photon was then detected by a single photon detector based on an InGaAs/InP avalanche photodiode. The coincidence signal between the two detectors was collected for various polarization combinations, which were governed by the two PAs.

From the polarization correlation measurements, we reconstructed the density matrix of the two-photon polarization state [13], [14]. Figure 3 shows the density matrix $\rho$ we thus obtained [15]. Here, the phase in (4) was set to be $\phi=0$; the expected state was

$$
\left|\Psi^{+}\right\rangle=\frac{1}{\sqrt{2}}\left(|\mathrm{H}\rangle_{1}|\mathrm{~V}\rangle_{2}+|\mathrm{V}\rangle_{1}|\mathrm{H}\rangle_{2}\right) .
$$

The fidelity $F$ of the reconstructed density matrix $\rho$ to the ideal Bell state (5) was $F=\left\langle\Psi^{+}|\rho| \Psi^{+}\right\rangle=0.94$, indicating that the generated state was close to the ideal Bell state.

Our photon pair source also produces frequencyentangled state

$$
|\Psi\rangle=\frac{1}{\sqrt{2}}\left(\left|\omega_{1}\right\rangle_{\mathrm{H}}\left|\omega_{2}\right\rangle_{\mathrm{V}}+e^{i \phi}\left|\omega_{2}\right\rangle_{\mathrm{H}}\left|\omega_{1}\right\rangle_{\mathrm{V}}\right)
$$

when we divide the photons in terms of their polarization. The phase $\phi$ is the same as that in (4) and is controlled by the interferometer as described above. In this case, the non-degenerate Hong-Ou-Mandel (NDHOM) interference [16] exhibits the beat-note fringes expressed by $1-\cos \phi$. The fringe visibility reflects the coherence between the two terms in the state (6), and thus manifests the frequency entanglement. To observe the ND-HOM interference, we replaced the dichroic mirror in Fig. 2 with a polarized beamsplitter (a)

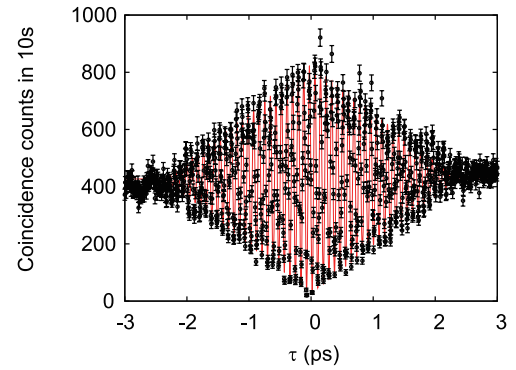

(b)

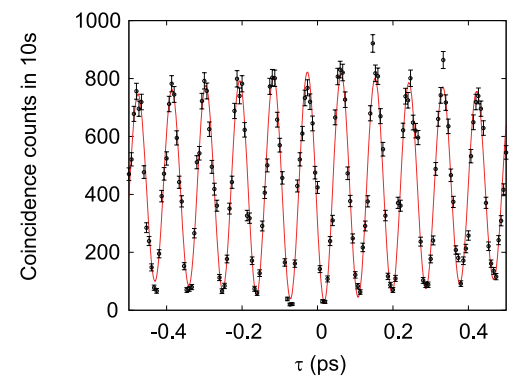

Fig. 4 Observed ND-HOM interference as a function of $\tau$ $=\Delta L / c$. Figure (b) is the enlarged view of (a) around $\tau \sim 0$.

(PBS) preceded by a half wave plate to divide the photons in terms of $\pm 45^{\circ}$ polarizations. The observed NDHOM interference (Fig. 4) [17] exhibited the high visibility $(93.4 \pm 1.9 \%)$ fringes, indicating that the photon pair is in the frequency-entangled state (6). The envelope of the fringe visibility shown in Fig. 4 (a) reflects the temporal overlap between the photon wave packets. We note that the photon pair state (6) generated by this method can be regarded as a state having controlled frequency correlation in the two discrete frequencies, i.e., two frequency bins.

\section{Controlled frequency correlation us- ing EPM}

In usual phase matching methods including QPM, the phase matching only at a certain frequency is taken into account. To control the spectrotemporal properties of the twin photons generated by SPDC, one must consider the deviation of the phase matching condition around the frequency concerned. The technique is called as EPM. Assume that the phase matching condition (2) or (3) is fulfilled at $\omega_{s}, \omega_{i}$ and $\omega_{p}=\omega_{s}+\omega_{i}$ and that the pump, signal, and idler photons propagate collinearly. Define the wavenumber deviation $\Delta k$ from the phase-matching condition as a function of $\omega_{s}$ and $\omega_{i}$ as

$$
\begin{aligned}
\Delta k\left(\omega_{s}, \omega_{i}\right)= & k_{s}\left(\omega_{s}\right)+k_{i}\left(\omega_{i}\right) \\
& -k_{p}\left(\omega_{s}+\omega_{i}\right)+m \frac{2 \pi}{\Lambda} .
\end{aligned}
$$




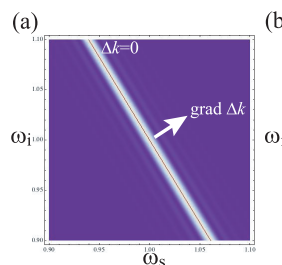

$\omega_{\mathrm{s}}^{\mathrm{in}}$

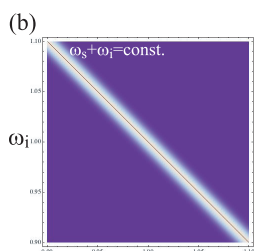

$\omega_{\mathrm{s}}$

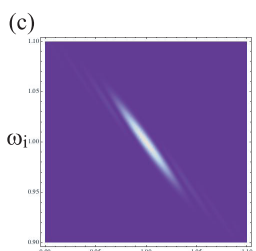

$\omega_{\mathrm{s}}$
Fig. 5 Example of (a) phase-matching function $f$, (b) pump spectrum $g$, and (c) joint spectral distribution $f g$.

The photon flux of the SPDC is proportional to the phase-matching function

$$
f=\left(\frac{\sin \Delta k L / 2}{\Delta k / 2}\right)^{2},
$$

where $L$ is the crystal length. Figure 5 (a) is the example of the two-dimensional density plot of $f$ as a function of $\omega_{s}$ and $\omega_{i}$. The ridge of $f$ exhibits the direction along which the phase-matching condition $\Delta k=0$ is fulfilled. The direction perpendicular to the ridge is expressed by the direction of the gradient

$$
\begin{aligned}
\operatorname{grad} \Delta k= & \left(\frac{\partial \Delta k}{\partial \omega_{s}}, \frac{\partial \Delta k}{\partial \omega_{i}}\right) \\
= & \left(v_{g}^{-1}\left(\omega_{s}\right)-v_{g}^{-1}\left(\omega_{p}\right),\right. \\
& \left.v_{g}^{-1}\left(\omega_{i}\right)-v_{g}^{-1}\left(\omega_{p}\right)\right) .
\end{aligned}
$$

Here, $v_{g}=d \omega / d k$ is the group velocity. Thus, the gradient direction is governed by the dispersion of refractive indices. In addition, the energy conservation condition (1) must be fulfilled in the SPDC. This means that the SPDC flux is also proportional to the power spectrum of the pump beam $g\left(\omega_{p}\right)=g\left(\omega_{s}+\omega_{i}\right)$, an example of which is shown in Fig. 5 (b). Thus the total SPDC photon flux is proportional to the product of $f$ and $g$, as shown in Fig. 5 (c). The two-photon state of the SPDC can be expressed by

$$
|\Psi\rangle=\iint d \omega_{s} d \omega_{i} S\left(\omega_{s}, \omega_{i}\right)\left|\omega_{s}\right\rangle\left|\omega_{i}\right\rangle .
$$

The "joint spectral distribution" $f g$ of the SPDC is proportional to $\left|S\left(\omega_{s}, \omega_{i}\right)\right|^{2}$.

Here, we consider the three special cases:

(i) $v_{g}^{-1}\left(\omega_{s}\right)=v_{g}^{-1}\left(\omega_{i}\right)$,

(ii) $v_{g}^{-1}\left(\omega_{s}\right)+v_{g}^{-1}\left(\omega_{i}\right)=2 v_{g}^{-1}\left(\omega_{p}\right)$,

(iii) $v_{g}^{-1}\left(\omega_{s}\right)=v_{g}^{-1}\left(\omega_{p}\right)$.

In these cases, the ridges of the joint spectral distribution are along $-45^{\circ},+45^{\circ}$, and $0^{\circ}$ in the $\omega_{s}-\omega_{i}$ plane, respectively. In cases (i) and (ii), and in the limit of long crystal, the two-photon state is entangled with anti-correlated and correlated frequencies,

$$
\left|S\left(\omega_{s}, \omega_{i}\right)\right|=\delta\left(\omega_{s}+\omega_{i}\right),
$$

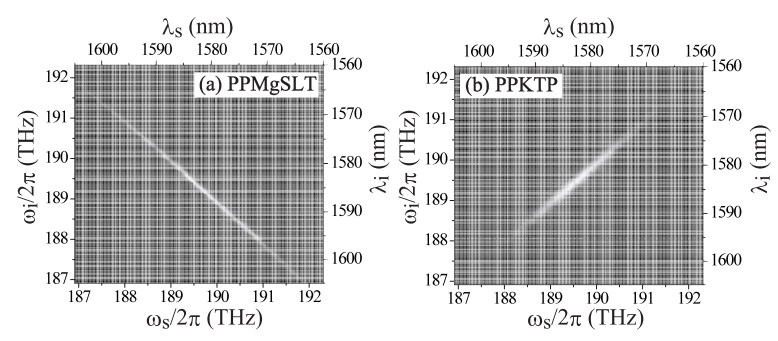

Fig. 6 Measured joint spectral distributions of SPDC generated from (a) PPMgSLT and (b) PPKTP.

$$
\left|S\left(\omega_{s}, \omega_{i}\right)\right|=\delta\left(\omega_{s}-\omega_{i}\right) g^{1 / 2}\left(\omega_{s}+\omega_{i}\right),
$$

respectively. In case (iii), the two-photon state is a product (separable) state with no frequency correlation

$$
\left|S\left(\omega_{s}, \omega_{i}\right)\right|=\delta\left(\omega_{i}-\omega_{0}\right) g^{1 / 2}\left(\omega_{s}+\omega_{0}\right),
$$

where $\omega_{0}$ is the center frequency of the idler. This state is useful in the application of heralded single photon generation; the single signal photon heralded by the idler is spectrally pure because of the lack of frequency correlation between the signal and idler photons. In this context, a number of works have been carried out to generate spectrally pure, heralded single photons using SPDC [4], [5], [18] and four-wave mixing in optical fibers [19]-[22].

We have generated and demonstrated the three kinds of two-photon states. For the state in case (i), we used periodically poled $\mathrm{MgO}$-doped stoichiometric $\mathrm{LiTaO}_{3}$ (PPMgSLT) in the type-II QPM condition. For the state in case (ii), we used periodically poled $\mathrm{KTiOPO}_{4}$ (PPKTP) crystal in the type-II QPM. We used a modelocked Ti:sapphire laser operated in cw mode as a pump source for the PPMgSLT, and in pulsed mode (spectral width: $6 \mathrm{~nm}$, pulse duration: $120 \mathrm{fs}$ ) for the PPKTP crystal. The observed joint spectral distributions for PPMgSLT and PPKTP are shown in Fig. 6 (a) and (b), respectively [23]. We see that the two-photon spectra exhibit the frequency anti-correlation (11) and correlation (12), as expected.

In case (ii), the widths of the joint spectral distribution along $-45^{\circ}$ and $+45^{\circ}$ are governed by the widths of the phase-matching function $f$ and the pump spectrum $g$, respectively. Thus, we can independently control the widths along the two orthogonal directions. In particular, when the two widths are identical, we can make a circular joint spectral distribution. The SPDC in this case has no spectral correlation between signal and idler photons, providing high spectral purity of each photon and high indistinguishability between photons generated from independent SPDC sources. The photons produced in this way are very useful in generating various quantum states consisting of multiple number 


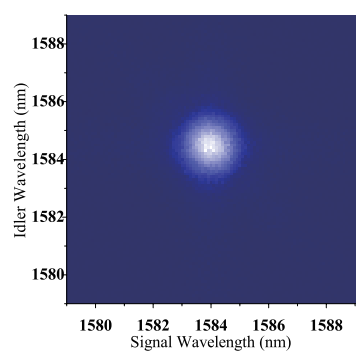

Fig. 7 Joint spectral distribution of SPDC generated from a 30-mm-long PPKTP crystal and 0.4-nm-wide pump pulses. (a)

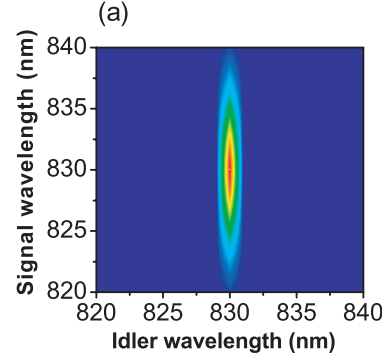

(b)

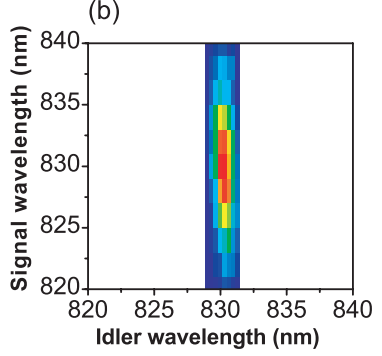

Fig. 8 (a) Calculated and (b) measured joint spectral distributions of SPDC generated from KDP.

of photons, since such photons must be indistinguishable with each other. Figure 7 presents the circularshaped joint spectral distribution thus generated, using a 30-mm-long PPKKTP and 0.4-nm-wide pump pulses (temporal duration: $3 \mathrm{ps}$ ) at $\lambda=792 \mathrm{~nm}$ [24]. We see that the joint spectral distribution has a good circular shape, as expected. It is also noteworthy that the twophoton generation rate $(\eta)$ of this method was quite high: the observed $\eta=4.1 \times 10^{4} \mathrm{~s}^{-1} \mathrm{~mW}^{-1}$ are almost one order of magnitude grater than those obtained by existing techniques to produce indistinguishable photon pairs using SPDC.

To generate the state in case (iii), we used $\mathrm{KH}_{2} \mathrm{PO}_{4}$ (KDP) crystal in type-II QPM pumped at $\lambda=415 \mathrm{~nm}$. The resulting SPDC centered at $\lambda=830 \mathrm{~nm}$ has a joint spectral distribution as in (13) [4], [5]. We used a modelocked Ti:sapphire laser (spectral width: $7.1 \mathrm{~nm}$, pulse duration: $100 \mathrm{fs}$ ) as a pump source. Figures 8 (a) and (b) present the calculated and observed joint spectral distributions, respectively. The observed joint spectral distribution is in good agreement with the calculation, showing the characteristic profile in (13). The Schmidt Value [5] calculated from the joint spectral distribution is 1.03 , which ensures high purity of the state. HongOu-Mandel (HOM) interference [25] is a good test to check the indistinguishability between single photons. In fact, Mosley et al. demonstrated that the signal (or idler) photons generated from the two independent (a)

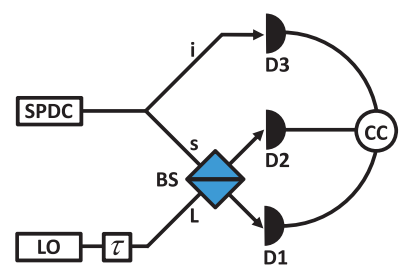

(b)

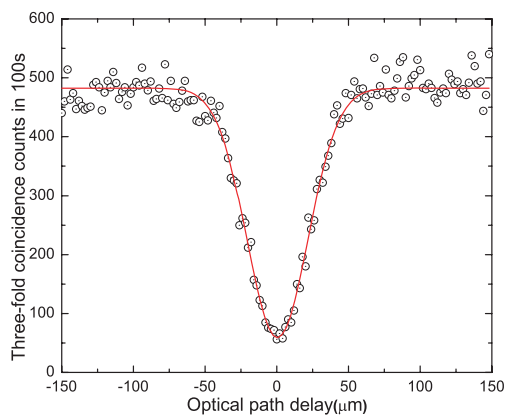

Fig. 9 (a) Sketch of the Hong-Ou-Mandel interference experiment between the signal (s) and coherent (L) photons heralded by the idler (i) photon. Note that the interference was observed upon three-fold coincidence between the signal, idler, and coherent photons. (b) Observed Hong-OuMandel interference.

SPDC sources exhibited the high-visibility HOM interference [4], [5]. Also interesting is that the signal photon thus generated has a pure spectrotemporal mode with a bandwidth that is almost the same as that of the coherent photons generated from the laser source, the second harmonic of which is the pump source of the SPDC. In this context, the signal photon heralded by the idler should also exhibit the HOM interference with a weak coherent light from the laser source [18], [26]. As shown in Fig. 9, we have observed [27] the highvisibility HOM interference manifesting the high spectral purity of the signal photon that is indistinguishable from the coherent photons. The observed visibility $89.4 \pm 0.5 \%$ was the highest in the HOM interference between single and coherent photons ever reported without spectral filtering [18], [26]. We also note that our pure, heralded single photon source is much more efficient than those employing spectral filtering [26]. Thus, our heralded single photon source will be useful in generating multiple photons necessary for various experiments in multi-qubit QICT. Also, its ability of high-visibility interference with coherent photons is promising for the new hybrid QICT protocols that combine discrete photon optics and continuous-variable optical techniques, e.g., homodyne detection.

\section{SUMMARY}

We have demonstrated the generation of polarization and frequency entangled photons using two-period 
QPM. Also demonstrated are the photon pair generation with controlled frequency correlation by use of EPM. These techniques are to be powerful tools in the development of advanced QICT.

\section{Acknowledgement}

This work was supported in part by a Grant-in-Aid for Creative Scientific Research (17GS1204) of the Japan Society for the Promotion of Science.

\section{References}

[1] K. Edamatsu, "Entangled photons: generation, observation, and characterization," Jpn. J. Appl. Phys., vol.46, no.11, pp.7175-7187, 2007.

[2] S. Tanzilli, W. Tittel, H. D. Riedmatten, H. Zbinden, P. Baldi, M. DeMicheli, D. B. Ostrowsky, and N. Gisin, "PPLN waveguide for quantum communication," Eur. Phys. J. D, vol.18, no.2, pp.155-160, 2002.

[3] O. Kuzucu, M. Fiorentino, M. A. Albota, F. N. C. Wong, and F. Kärtner, "Two-photon coincident-frequency entanglement via extended phase matching," Phys. Rev. Lett., vol.94, 083601, Mar 2005.

[4] P. J. Mosley, J. S. Lundeen, B. J. Smith, P. Wasylczyk, A. B. U'Ren, C. Silberhorn, and I. A. Walmsley, "Heralded generation of ultrafast single photons in pure quantum states," Phys. Rev. Lett., vol.100, 133601, Jan 2008.

[5] P. J. Mosley, J. S. Lundeen, B. J. Smith, and I. A. Walmsley, "Conditional preparation of single photons using parametric downconversion: a recipe for purity," New J. Phys., vol.10, 093011, Jan 2008.

[6] E. Knill, R. Laflamme, and G. Milburn, "A scheme for efficient quantum computation with linear optics," $\mathrm{Na}$ ture, vol.409, pp.46-52, Jan 2001. 10.1038/35051009.

[7] R. Raussendorf, D. Browne, and H. Briegel, "Measurement-based quantum computation on cluster states," Phys Rev A, vol.68, 022312, Aug 2003.

[8] P. Walther, K. Resch, T. Rudolph, E. Schenck, H. Weinfurter, V. Vedral, M. Aspelmeyer, and A. Zeilinger, "Experimental one-way quantum computing," Nature, vol.434, pp.169-176, Mar 2005. 10.1038/nature03347.

[9] A. Yoshizawa and H. Tsuchida, "Generation of polarization-entangled photon pairs at $1550 \mathrm{~nm}$ using two PPLN waveguides," Electron. Lett., vol.39, no.7, pp.621-622, 2003.

[10] M. Hobden and J. Warner, "The temperature dependence of the refractive indices of pure lithium niobate," Physics Letters, vol.22, no.3, pp.243-244, 1966.

[11] D. H. Jundt, "Temperature-dependent Sellmeier equation for the index of refraction, ne, in congruent lithium niobate," Opt. Lett., vol.22, no.20, pp.1553-1555, 1997.

[12] S. Nagano, A. Syouji, R. Shimizu, K. Suizu, H. Ito, and K. Edamatsu, "Generation of cross-polarized photon pairs via type-ii third-order quasi-phase matched parametric down-conversion," Jpn. J. Appl. Phys., vol.48, no.5, 050205, 2009.

[13] D. F. V. James, P. G. Kwiat, W. J. Munro, and A. G. White, "Measurement of qubits," Phys. Rev. A, vol.64, 052312 , Oct 2001.

[14] A. G. White, D. F. V. James, W. J. Munro, and P. G. Kwiat, "Exploring hilbert space: Accurate characterization of quantum information," Phys. Rev. A, vol.65, 012301, Dec 2001.

[15] W. Ueno, F. Kaneda, H. Suzuki, S. Nagano, A. Syouji, R. Shimizu, K. Suizu, and K. Edamatsu, unpublished.

[16] Z. Y. Ou and L. Mandel, "Observation of spatial quantum beating with separated photodetectors," Phys. Rev. Lett., vol.61, pp.54-57, Jul 1988.

[17] F. Kaneda, H. Suzuki, R. Shimizu, S. Nagano, A. Syouji, K. Suizu, and K. Edamatsu, unpublished.

[18] K. Laiho, K. N. Cassemiro, and C. Silberhorn, "Producing high fidelity single photons with optimal brightness via waveguided parametric down-conversion," Opt. Express, vol.17, no.25, pp.22823-22837, 2009.

[19] K. Garay-Palmett, H. J. McGuinness, O. Cohen, J. S. Lundeen, R. Rangel-Rojo, A. B. U'Ren, M. G. Raymer, C. J. McKinstrie, S. Radic, and I. A. Walmsley, "Photon pair-state preparation with tailored spectral properties by spontaneous four-wave mixing in photonic-crystal fiber," Opt. Express, vol.15, no.22, pp.14870-14886, 2007.

[20] O. Cohen, J. S. Lundeen, B. J. Smith, G. Puentes, P. J. Mosley, and I. A. Walmsley, "Tailored photon-pair generation in optical fibers," Phys. Rev. Lett., vol.102, 123603, Jan 2009.

[21] M. Halder, J. Fulconis, B. Cemlyn, A. Clark, C. Xiong, W. J. Wadsworth, and J. G. Rarity, "Nonclassical 2-photon interference with separate intrinsically narrowband fibre sources," Opt. Express, vol.17, no.6, pp.4670-4676, 2009.

[22] C. Söller, B. Brecht, P. J. Mosley, L. Y. Zang, A. Podlipensky, N. Y. Joly, P. S. J. Russell, and C. Silberhorn, "Bridging visible and telecom wavelengths with a single-mode broadband photon pair source," Phys. Rev. A, vol.81, 031801, Mar 2010.

[23] R. Shimizu and K. Edamatsu, "High-flux and broadband biphoton sources?with controlled frequency entanglement," Opt. Express, vol.17, no.19, pp.16385-16393, 2009.

[24] M. Yabuno, R. Shimizu, Y. Mitsumori, H. Kosaka, and K. Edamatsu, unpublished.

[25] C. K. Hong, Z. Y. Ou, and L. Mandel, "Measurement of subpicosecond time intervals between two photons by interference," Phys. Rev. Lett., vol.59, pp.2044-2046, Nov 1987.

[26] J. G. Rarity, P. R. Tapster, and R. Loudon, "Nonclassical interference between independent sources," 
Journal of Optics B: Quantum and Semiclassical Optics, vol.7, no.7, p.S171, 2005.

[27] R.-B. Jin, J. Zhang, R. Shimizu, N. Matsuda, Y. Mitsumori, H. Kosaka, and K. Edamatsu, "High-visibility nonclassical interference between pure heralded single photons and weak coherent photons," arXiv, quant$\mathrm{ph} / 1010.5638$.

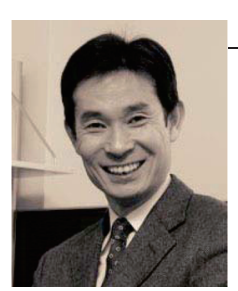

\section{Keiichi EDAMATSU}

Keiichi EDAMATSU is a professor of Research Institute of Electrical Communication, Tohoku University. He received B.S., M.S., and D.S. degrees in Physics from Tohoku University. He was a research associate in Department of Applied Physics, Faculty of Engineering, Tohoku University, a visiting associate in Norman Bridge Laboratory of Physics, California Institute of Technology, and an associate professor in Division of Materials Physics, Graduate School of Engineering Science, Osaka University. He is a member of the Physical Society of Japan and Optical Society of America. His current research interests are quantum optics, solid-state photophysics, and quantum information/communication science.

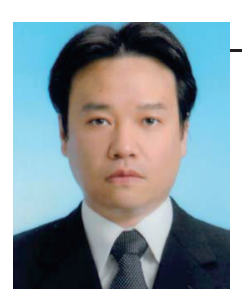

\section{Ryosuke SHIMIZU}

Ryosuke SHIMIZU was working at Research Institute of Electrical Communication, Tohoku University, as a research scientist of PRESTO, Japan Science and Technology Agency. He is currently an associate professor of the University of Electro-Communication.

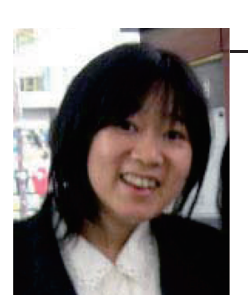

\section{Wakana UENO}

Wakana UENO is a doctoral student of Research Institute of Electrical Communication, Tohoku University.

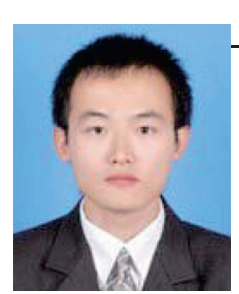

\section{Rui-Bo JIN}

Rui-Bo JIN is a doctoral student of Research Institute of Electrical Communication, Tohoku University.

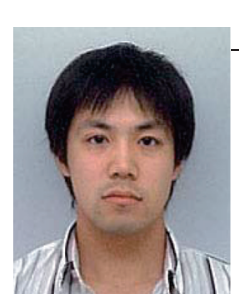

\section{Fumihiro KANEDA}

Fumihiro KANEDA is a doctoral student of Research Institute of Electrical Communication, Tohoku University.

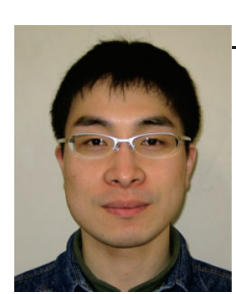

\section{Masahiro YABUNO}

Masahiro YABUNO is a doctoral student of Research Institute of Electrical Communication, Tohoku University.

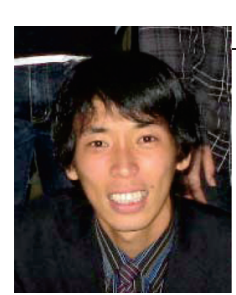

Hirofumi SUZUKI

Hirofumi SUZUKI was a master course student of Research Institute of Electrical Communication, Tohoku University. He is now belonging to Suzuki Motor Co.

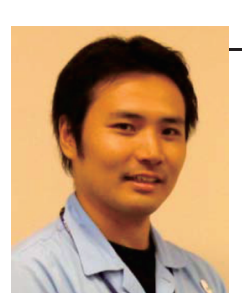

Shigehiro NAGANO

Shigehiro NAGANO was working at Research Institute of Electrical Communication, Tohoku University, as a postdoctoral fellow. He is now belonging to Sumitomo Electric Co. 


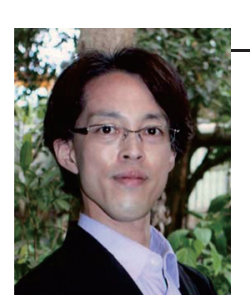

Atsushi SYOUJI

Atsushi SYOUJI was working at Research Institute of Electrical Communication, Tohoku University, as a postdoctoral fellow. He is currently an assistant professor of University of Yamanashi.

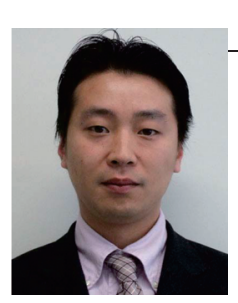

\section{Koji SUIZU}

Koji SUIZU is an assistant professor of Graduate School of Engineering, Nagoya University. He was working at Research Institute of Electrical Communication, Tohoku University, as an assistant professor. 